\title{
Consideration on sampling interval in Computer-generated hologram using angle spectrum method
}

\author{
Koki Nakashimaa,, ${ }^{\mathrm{a}, *}$ Mizuho Kimura ${ }^{\mathrm{a}}$, Shiyuan Yang ${ }^{\mathrm{a}}$ \\ Kyushu Institute of Technology, 1-1 Sensui-cho Tobata-ku Kitakyushu-shi Fukuoka-ken, 804-8550, Japan \\ *Corresponding Author: ajgdb_254622_bskb13@yahoo.co.jp
}

\begin{abstract}
A hologram is a recording medium created by holography that records and reproduces a threedimensional object. Creating a hologram on a computer is Computer-generated holography, and a hologram created by computer holography is called a Computergenerated hologram.In this paper,the angular spectrum method which has short calculation time and can reproduce even in the short distance is used.Computergenerated hologram can be obtained by calculation on a computer, but the distribution of holograms is obtained by discrete values because computers can not handle continuous values. Therefore, sampling intervals must be taken into account. In this paper, we study and consider how the sampling interval affects when reproducing a hologram created by the angular spectrum method. As a result of the simulation, it was found that the pixel pitch has to be changed according to the distance of reproducing the hologram
\end{abstract}

Keywords: Computer-generated hologram, angular spectrum method,

\section{Introduction}

Since images such as photographs recorded only the amplitude information of the original object light, information on the optical path can not be obtained. However, the hologram records not only the amplitude information of the original object light but also the phase information. It is possible to reproduce the stereoscopic image because the phase information is information on the optical path. Although information of interference fringes also has amplitude information and phase information, it is difficult to record these two pieces of information..However, since each amplitude or phase information of the interference fringes contains both the amplitude and the phase information of the object light, only either the amplitude or the phase of the interference fringe can be recorded. Generally,the recording material mostly reacts to the amplitude information of the light.Therefore amplitude information of interference fringes is often recorded. A hologram recorded with amplitude information as described above is called an amplitude hologram. On the other hand, a hologram recording phase information is called a phase hologram. The amplitude hologram reproduces the original three-dimensional object by amplitude modulation and the phase hologram reproduces by phase modulation. Holography can be divided into two processes: a process of creating a hologram and a process of reproducing a hologram. Creating a hologram on a computer is Computergenerated holography, and a hologram created by Computer-generated holography is called a Computergenerated hologram. This technology was invented by Roman et al in 1966. Like an optical hologram, a hologram for reproducing a three-dimensional object by amplitude modulation is called an amplitude type Computer-generated hologram, and a hologram for reproducing a three-dimensional object by phase modulation is called a phase type Computer-generated hologram. Computer-generated holography can also make wavefronts with various amplitude and phase distributions, so holograms can be recorded for objects that can not exist. Therefore, various applications can be considered. The method of creating a Computergenerated hologram includes diffraction integration of Fresnel Kirchoff, Fresnel approximation, angular spectrum method, and the like. In the diffraction integral of Fresnel-Kirchhoff, the calculation time is very slow, and the hologram created by the Fresnel approximation can not be reproduced in the short distance. Therefore the calculation time is relatively 
short, and the angular spectrum method which can be reproduced even in the short distance is described in this paper. A Computer- generated hologram can be obtained by calculation on a computer, but because computers can not handle continuous values, the distribution of holograms is obtained by discrete values. Therefore, quantization error and discretization error occur. Also, sampling intervals must be taken into consideration when dealing with discrete values. In this paper, we study and consider how the sampling interval affects when reproducing a hologram created by the angular spectrum method.

\section{Computer-generated hologram}

\section{1 angular spectrum method}

The angular spectral method is one of methods of creating Computer-generated holograms. Figure. 1 shows a calculation method of the angular spectrum method. First, complex amplitude distribution of the original image is input. Second, the distribution is Fourier transformed. Third,calculate the diffraction phase of the angular spectrum and add it to the distribution of the Fourier transform plane. Finally,the distribution obtained by adding the phase components.isinverse fourier transformed. The distribution created by inverse Fourier transformation is a hologram made by angular spectrum method.

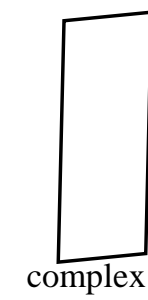

amplitude distribution

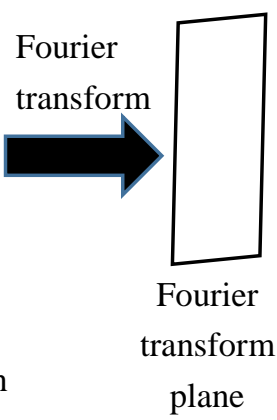$$
\text { invers }
$$

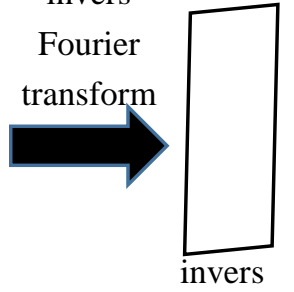

Fourier transform plane.

Fig.1. angular spectrum method

\subsection{Principle of Computer-generated hologram}

a Computer-generated hologram is a method of generating a hologram on a computer. Computer hologram calculates diffraction of object light and reference light and distribution of interference fringes by calculation. Therefore, there is an advantage that the object light can handle even imaginary things that can not exist in the real world. All data that can be handled on the computer are discrete values. Therefore, in the case of Computer-generated holograms, errors due to discretization and quantization occur. In order to make this error as small as possible, it suffices to obtain a sufficiently large number of discrete points. Taking a large number of discrete points in a Computergenerated hologram corresponds to making the size of the hologram sufficiently large in the optical hologram. However, taking a sufficiently large number of discrete points in the Computer-generated hologram leads to an increase in calculation time. Also, it requires a lot of memory space to store data of discrete points.

\subsection{Complex amplitude hologram}

The complex amplitude hologram is a hologram that simultaneously records the amplitude and phase information of object light. Complex amplitude holograms are often used as simulations in Computergenerated holograms. In this paper, we consider the reconstructed image reproducing the hologram on the simulation and the sampling interval, so we create and reproduce a complex amplitude hologram.

\section{Sampling interval by the angular spectrum method}

\subsection{Determination of hologram by angle spectrum method}

In order to reproduce the hologram created with the angular spectrum, the pixel pitch of the hologram must be changed according to the distance. For that purpose, the following three equations are necessary.

$$
\begin{gathered}
N^{\prime} \geq N \\
\Delta x N=\Delta u N^{\prime} \\
\Delta u \leq \frac{\lambda z}{\Delta x N}
\end{gathered}
$$

Where $N$ and $N^{\prime}$ are the number of sampling points of the original image and the hologram, and $\Delta x$ and $\Delta u$ are the pixel pitch of the original image and the hologram. Also, $z$ is the distance, $\lambda$ is the wavelength, this time it is $632[\mu \mathrm{m}]$. Equation (1) is a formula required to be a complex amplitude hologram. 
Equation (2) is a formula that the hologram and the original image have the same size. Equation (3) is the sampling theorem. The original image $(\Delta x=10[\mu \mathrm{m}], N$ $=128[$ pixel] $)$ is shown in Fig2. When the above equation is satisfied, the $\mathrm{F}$ image reproduced with $z=$ $0.25,0.5,1.0,3.0[\mathrm{~m}]$ created in the angular spectrum is shown in Fig. 3(a)to(d). Fig.4(a)to(d) shows an image obtained by reproducing a hologram created using Fresnel approximation under the same conditions as the angular spectrum method.

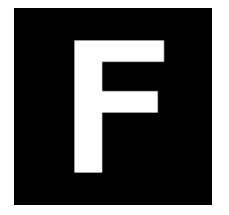

Fig.2. original image

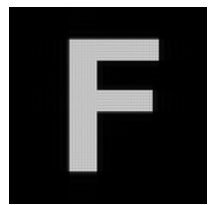

(a) $\mathrm{z}=0.25[\mathrm{~m}]$

$N^{\prime}=1024$ [pixel]

$\Delta u=12.5[\mu \mathrm{m}]$

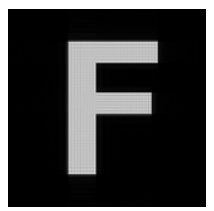

(c) $\mathrm{z}=1.0[\mathrm{~m}]$

$N^{\prime}=256[$ pixel]

$\Delta u=50.0[\mu \mathrm{m}]$

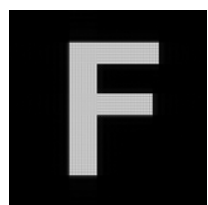

(b) $\mathrm{z}=0.5[\mathrm{~m}]$

$N^{\prime}=512$ [pixel]

$\Delta u=25.0[\mu \mathrm{m}]$

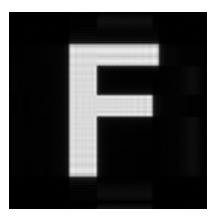

(d) $\mathrm{z}=3.0[\mathrm{~m}]$

$N^{\prime}=128$ [pixel]

$\Delta u=100.0[\mu \mathrm{m}]$

Fig.3. Comparison based on the distance of the image reproduced from the hologram created by the angular spectrum method

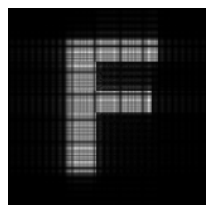

(a) $\mathrm{z}=0.25[\mathrm{~m}]$

$N^{\prime}=1024$ [pixel]

$\Delta u=12.5[\mu \mathrm{m}]$

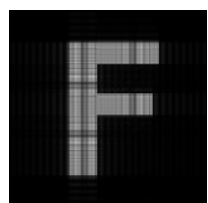

(b) $\mathrm{z}=0.5[\mathrm{~m}]$

$N^{\prime}=512[$ pixel]

$\Delta u=25.0[\mu \mathrm{m}]$
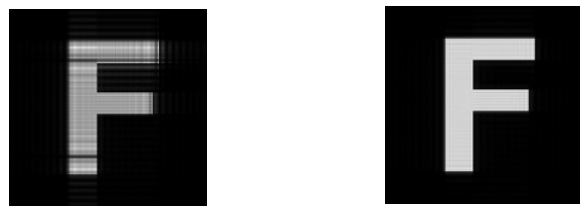

(c) $\mathrm{z}=1.0[\mathrm{~m}]$

$N^{\prime}=256[$ pixel]

$\Delta u=50.0[\mu \mathrm{m}]$ (d) $\mathrm{z}=3.0[\mathrm{~m}]$

$N^{\prime}=128$ [pixel]

$\Delta u=100.0[\mu \mathrm{m}]$
Fig.4. Comparison based on the distance of the image reproduced from the hologram created by Fresnel approximation

From Fig. 3 and 4 it can be seen that holograms created with Fresnel approximation can not be reproduced at short distances and holograms created with angular spectra can be reproduced at short distances, but the pixel pitch of the hologram must be changed.

\subsection{Relationship between distance and reproduction image error by angular spectral method}

In chapter 3.1 we changed the pixel pitch of the hologram, but the pixel pitch is fixed to one when optically reproducing the hologram. Therefore, fix the pixel pitch of the hologram and reproduce it with changing the distance. Therefore, fix the pixel pitch of the hologram to $20.0[\mu \mathrm{m}]$. Further, the number of sampling points of the original image is fixed at 128 [pixel] and the pixel pitch is fixed at $20.0[\mu \mathrm{m}]$. Fig 5. (a)to(d) show images obtained by reproducing the hologram created with the angular spectrum when the distances $\mathrm{z}=0.1,0.2,0.4,0.8[\mathrm{~m}]$.
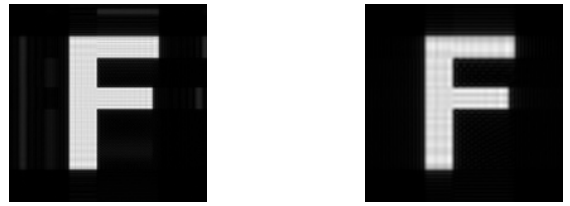

(a) $\mathrm{z}=0.1[\mathrm{~m}]$

$N^{\prime}=128$ [pixel]

$\Delta u=20[\mu \mathrm{m}]$ (b) $\mathrm{z}=0.2[\mathrm{~m}]$

$N^{\prime}=128$ [pixel]

$\Delta u=20[\mu \mathrm{m}]$ 


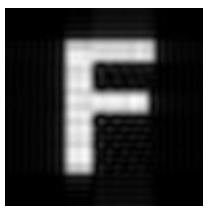

(c) $\mathrm{z}=0.4[\mathrm{~m}]$

$N^{\prime}=128$ [pixel]

$\Delta u=20[\mu \mathrm{m}]$

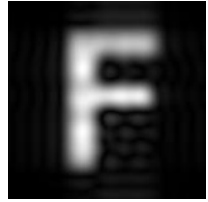

(d) $\mathrm{z}=0.8[\mathrm{~m}]$ $N^{\prime}=128$ [pixel]

$\Delta u=20[\mu \mathrm{m}]$

Fig.5. Images obtained by reproducing a hologram created with an angular spectrum when the pixel pitch of the hologram is fixed

From Fig.5,you can see that if the pixel pitch of the hologram is fixed, the error of the reconstructed image changes depending on the distance.

\section{Conclusions}

Therefore, when creating and reproducing a hologram by the angular spectrum method, the pixel pitch of the hologram must be changed according to the distance to reproduce. Considering the case of optical reproduction, although reproduction is possible when the pixel pitch of the hologram is fixed, an error occurs depending on the distance.

\section{References}

(1) P.Hariharan : "Holographic principle", Optronics campany, Vol. 1, No. 1, pp. 88-95, 2003

(2) Joseph W.Goodman: "What is Engineering", Morikita publisher, Vol. 3, No. 1, pp. 55-73, 2012

(3) Atsushi Matsumura: "Optical", Asakra bookstore, Vol. 1, No. 1, pp.86-94 1983

(4) George B.Arfken and Hans J.Weber : "Fourier transform and variational method", Koudann company, Vo4. 1, No. 4, pp.72-86, 2009

(5) Yokohiko Yatagai: "Light and Fourier Transform", Asakra bookstore, No. 6, pp.9-45,1997 